\title{
Arthroscopic Reconstruction of the Posterior Cruciate Ligament With Ligament Advanced Reinforcement System and Hamstring Tendon Autograft :A Retrospective Study
}

\author{
Yang Luo \\ Chinese PLA General Hospital \\ Zhigang Wang \\ Chinese PLA General Hospital \\ Zhijiang Li \\ Chinese PLA General Hospital \\ Min Wei ( $\square$ weimindr301@163.com ) \\ Chinese PLA General Hospital
}

\section{Research article}

Keywords: posterior cruciate ligament, LARS, Ligament Advanced Reinforcement System, hamstring tendon, reconstruction

Posted Date: July 22nd, 2020

DOI: https://doi.org/10.21203/rs.3.rs-44920/v1

License: (c) (i) This work is licensed under a Creative Commons Attribution 4.0 International License. Read Full License 


\section{Abstract}

Background Both Ligament Advanced Reinforcement System(LARS) and hamstring tendon autograft can serve as grafts for posterior cruciate ligament (PCL) reconstruction but few studies compared their differences.This study aimed to compare the clinical efficacy of arthroscopic reconstruction of PCL with LARS and hamstring tendon autograft.

Methods 36 patients who had received PCL reconstruction under arthroscopy were retrospectively analyzed. 15 patients received reconstruction using LARS (LARS group) and 21 using hamstring tendon autograft (HT group). The pre- and post-operative subjective scores and knee stability were evaluated.

Results 36 patients were followed up for a period of 2 to 10.5 years ( $4.11 \pm 2.0$ years on average). The last follow-up showed that functional scores and knee stability were all significantly improved in both groups $(P<0.05)$. Six months after operation, Lysholm scores and IKDC subjective scores were higher in LARS group than in HT group $(P<0.05)$. Nonetheless, the last follow-up showed no statistically significant differences in the functional scores and the posterior drawer test between the two groups $(P>0.05)$. In LARS group and HT group, 12 and 9 patients, respectively had KT1000 values less than $3 \mathrm{~mm}$, with the difference being statistically significant $(P<0.05)$. In $\mathrm{HT}$ group, the diameter of the four-strand hamstring tendon was positively correlated with height $(P<0.05)$, which was $7.37 \pm 0.52 \mathrm{~mm}$ in males and $6.50 \pm 0.77$ $\mathrm{mm}$ in females, and the difference was statistically significant $(P<0.05)$.

Conclusion Both LARS and hamstring tendon could achieve good clinical efficacy for PCL reconstruction खbut patients in LARS group had faster functional recovery and better knee stability. LARS is especially suitable for those who hope to resume activities as early as possible.

\section{Background}

Posterior cruciate ligament (PCL) is the major structure that limits the backward movement of tibia. Isolated PCL injury accounts for $3 \%$ of all knee ligament injuries $(1,2)$. The stability of knee joint of PCL injury is reduced, and it usually lead to pain, swelling and disfunction of knee. Those patients whose clinical symptoms persist after conservative treatment usually need surgical intervention.

Currently, arthroscopic reconstruction of PCL has been extensively used, with satisfactory results accomplished. The grafts principally include autologous or allogenic ligaments and artificial ligaments, such as ligament advanced reinforcement system ( LARS ). Hamstring tendon is the most commonly used graft, which is characterized by easy availability, freedom from rejection and no risk of infection ( 3 , 4). LARS was first used for ACL reconstruction and achieved excellent results. Since then, some researchers tried to use LARS to reconstruct $\operatorname{PCL}(5,6)$. However, few studies had compared the efficacy of LARS and hamstring tendon for the PCL reconstruction. Recently, Saragagliad et al (7) compared the efficacy of the two grafts but they performed open operation with a new method of fixation. In this study, we compared the clinical efficacy of arthroscopic PCL reconstruction with LARS and hamstring tendon autograft, on the hypothesis that the two grafts might yield similar results. 


\section{Materials And Methods}

\subsection{Criteria of inclusion and exclusion}

Upon the approval by the Ethics Committee of the PLA General Hospital, Beijing, China, this study retrospectively examined patients who had received arthroscopic PCL reconstruction with LARS and hamstring tendon autograft from July, 2009 to June, 2018.

Inclusion criteria: (1) PCL injuries (grade II or III) diagnosed by MRl; (2) age range: 16-60 years; (3) having no injuries of other peri-knee ligaments or having injuries of other ligaments but no need for surgical reconstruction; (4) followed up for over two years.

Exclusion criteria: (1) Patients had received reconstruction of other ligaments at the same time; (2) associated peri-knee fracture or vascular injuries; (4) accompanied by severe osteoarthritis; (5) history of ipsilateral or contralateral knee surgery ; (5) Imaging data were not complete.

\subsection{Patient Data}

A total of 36 patients were included. 15 patients received PCL reconstruction with LARS (LARS group) and 21 patients with hamstring tendon (HT group). Written informed consent was obtained from each single participant and the reconstruction procedures were performed by the doctors of the same surgeon team. Their clinical data are as follows:

The LARS group contained ten males and five females, with their age ranging from 18 to 57 years (mean: $39.7 \pm 12.5$ years). Seven patients had injuries of meniscus or cartilage and four had injuries of other ligaments. Among them, two had ACL injury, one had LCL injury and one had MCL injury.

The HT group had 15 males and 6 females and they were aged 16-60 years (mean age: $36.5 \pm 13.1$ years). Nine patients had accompanying injuries of meniscus or cartilage and three suffered from injuries of other ligaments. Among them, one had MCL injury and two had LCL injury.

Clinical assessment turned out that there was no need for surgical treatment for these injuries of other ligaments. No statistically significant differences were found in gender, age, height, weight, BMI, injured sides and accompanying injuries between the two groups ( $P>0.05)$ (Table 1).

\subsection{Surgical technique}

A standard arthroscopy was performed in all patients to allow inspection and evaluation the construcion of the joint. The injury of PCL and integrity of other ligaments were confirmed. Then the associated injuries of meniscus and cartilage were dealed with meniscoplasty and chondroplasty.

In the LARS group, tibial and femoral tunnels were reamed by employing a $6.0 \mathrm{~mm}$ cannulated drill using anteromedial portal technique (8). A $6.0 \mathrm{~mm}$ LARS was introduced from the tibial tunnel into the femoral tunnel. The femoral side of the ligament was fixed by an interface screw. At keen flexion of $90^{\circ}$, an 
assistant adjusted the tension of the PCL by conducting the anterior drawer test. Then the tibial side of ligament was fixed by an interface screw. (Figure. 1).

In the HT group, hamstring tendon was harvested using conventional technique(8). The entire tendons were folded in half and looped on a titanium button(SmithNephew, EndoButton) to result in the fourstrand, with their diameters and length measured. Tibial and femoral tunnels were reamed using anteromedial portal technique(8). At knee flexion of $90^{\circ}$, an assistant adjusted the tension of the PCL by conducting the anterior drawer test. Then the tibial side of graft was fixed by an interface screw (SmithNephew) (Figure.2).

\subsection{Post-operative rehabilitation}

In the LARS group, the knee was put in full extension with braces. On the first post-operative day, patients could do active ROM exercise. After two weeks postoperatively, patients were allowed to walk short distance with partial weight-bearing under the protection of braces and crutches. During the fourth to sixth week after operation, the patients could walk with crutches with full weight-bearing. In the seventh week after operation, the braces could be removed, and daily activities were resumed. In the third month after operation, the patients could gradually perform some low-intensity sports activities.

In the HT group, the knee was put in full extension with braces and the patients were allowed to perform isometric contraction of knee. For each ten days after operation, the flexion range was increased by the interval of $30^{\circ}$. In the fifth week after operation, patients could walk under the protection of crutches and braces, with partial weight-bearing. In the ninth week after operation, patients could gradually walk on full weight-bearing. In the fourth month after operation, the crutches and braces were removed and the patients could engage in the daily activities. During the fifth to sixth month after operation, the patients could take part in low-intensity sports activities.

\subsection{Evaluation}

Lysholm scores and IKDC subjective scores were used for functional evaluation of knee joint before and 6, 12 months after operation and at the last follow-up. Tegner activity scores was employed to rate the level of activity of patients before the operation and at the last follow-up. Posterior drawer test and KT 1000 device measurement $\left(90^{\circ}\right.$ flexion and $\left.132 \mathrm{~N}\right)(9)$ were performed to assess knee stability. As compared with the contralateral knee, the posterior drawer test rates the laxity in four degrees, with no laxity, side-to-side differences less than $5 \mathrm{~mm}$, between $5-10 \mathrm{~mm}$ and greater than $10 \mathrm{~mm}$ listed as grade $0,1,2$ and 3, respectively. Knee stability was rated "good" when the side to side differences of KT1000 were less than $3 \mathrm{~mm}(4,9,10)$.

The diameters of four-strand hamstring grafts were measured in 21 patients in HT group and their correlation with height, weight, and BMI were analyzed. The differences in the diameter of hamstring tendons were compared between the two gender groups.

\subsection{Statistical analysis}


The data were analyzed with SPSS 22.0 software. The chi-square test was used for categorical variables, and an unpaired Student's $t$ test was used for the comparisons of continuous data when the data were normally distributed. The Wilcoxon signed rank test was used when the data were in non-normal distribution. The Correlation analysis were assessed by Spearman correlation test. Statistical significance was established at $P$ value $<0.05$.

\section{Results}

\subsection{Operation related complications and follow-up}

All the patients had no serious complications such as infection, vascular or nerve injury. One patient developed relatively severe pain in harvest area and relieved pain by symptomatic treatment. The 36 patients were followed up for an average of $4.11 \pm 2.0$ years(range: 2-10.5 years). One patient in LARS group and four patients in HT group, respectively, still had the sense of knee instability but no patient required revision.

\subsection{Pre- and post-operative knee function and stability}

The last follow-up revealed that the Lysholm scores, IKDC scores and Tegner activity scores were all substantially improved compared with those before operation in both groups $(P<0.05)$. Posterior drawer test and KT1000 measurement also exhibited that the post-operative results were better than the preoperative ones $(\mathrm{P}<0.05)$ (Table 2$)$.

\subsection{Knee function and stability in the two groups}

No statistically significant differences were found in the results of pre-operative function scores and stability $(P>0.05)$. In the post-operative six months, the Lysholm scores and IKDC scores were higher in LARS group than in HT group $(P<0.05)$ but after 12 months operatively, the differences were not significant $(P>0.05)$. The last follow-up showed that there existed no statistically significant differences in function scores and the posterior drawer test between the two groups $(P>0.05)$. But the difference of KT1000 measurement was statistically significant. $(P<0.05)$ (Table 2 and Table 3 ).

\subsection{Correlation of the diameter of hamstring tendon}

In the HT group, the diameter of four-strand hamstring tendon was $7.11 \pm 0.69 \mathrm{~mm} \otimes$ range $₫ 5-8 \mathrm{~mm} \rrbracket$. The diameter was positively correlated with height $(r=0.619 \otimes P<0.05)$, but bore no relationship with weight and BMI. The diameter was $7.37 \pm 0.52 \mathrm{~mm}$ (range $\llbracket 6.5-8 \mathrm{~mm}$ ) for males, and $6.50 \pm 0.77 \mathrm{~mm}($ range: $5-7 \mathrm{~mm}$ ) for females, the difference was statistically significant $(P<0.05)$.

\section{Discussion}

Arthroscopic PCL reconstruction has become a principal alternative for the management of PCL injury but it remains controversial in many ways. One dispute concerns the selection of grafts. Hamstring 
tendon represents the most common graft used for PCL reconstruction $(3,4)$ since it is easily available and rejection-free, involves no risk of infection and can achieve quick tendon-bone healing. LARS mimics natural ligament in terms of biological properties (11). It possess excellent biocompatibility and its porous structure can stimulate and support ingrowth of collagen tissue. Moreover, the preserved remnant of the ligament is conductive to the ingrowth of tissues $(12,13)$. LARS was initially used for the reconstruction of ACL and then some researchers used it to construct PCL and attained satisfactory results. John Gliatis et al (6) utilized LARS for reconstruction of PCL in 31 patients. In a follow-up study lasting for 9.27 years on average, their last follow-up revealed a mean IKDC score of up to 79.32. Chen et al (5) used double-strand LARS for PCL reconstruction, and after two years operatively the average Tegner activity scores were improved to 6.0 from pre-operative 3.4 and the average Lysholm scores were raised to 91.76 from pre-operative 70.06 .

Both LARS and hamstring tendon autograft can serve as grafts for PCL reconstruction but few studies compared their differences and the currently available studies yielded inconsistent results. Li et al (14) found that Lysholm scores, Tegner activity scores, IKDC scores and knee joint stability of LARS group were better than hamstring group during two-year follow-up. The last follow-up of Xu et al (9) failed to show any statistically significant differences in the clinical results between the LARS group and hamstring group, including subjective function scores and ligament laxity.

In our series, the last follow-up showed that, after PCL reconstruction, subjective function scores were all conspicuously improved as compared with pre-operation, suggesting that the two grafts could substantially improve the activity level of patients. Our results were coincident with the findings of aforementioned studies. After six months operatively, the Lysholm scores, IKDC scores were higher in LARS group $(P<0.05)$, indicating that patients recovered more quickly in LARS group and could resume normal life earlier but such difference was not statistically significant after 12 months operatively $(P>$ 0.05). The last follow-up showed that there were no significant differences in all function scores $(P>$ $0.05)$ while the two groups respectively have 12 and nine patients whose KT-1000 was less than $3 \mathrm{~mm}(P$ $<0.05)$, indicating that patients in LARS group have better knee joint stability. Nonetheless, the posterior drawer test showed no statistically significant difference between the two groups.

In our study, LARS group outdid HT group in terms of knee stability. This, we believe, might be attributed to the inherent features of hamstring tendons autograft. Firstly, the PCL reconstruction with hamstring tendon involves tendon-bone healing, and the strength of insertion would reduced because it could not restored to normal tissue structure (15). Secondly, hamstring tendon autograft has to go through revascularization, cellular proliferation and remodeling to achieve "re-tendonization"(16), which takes one year to complete. During this process, the graft is subject to laxity. Thirdly, due to the gravitational force on the calf and pull of the hamstring muscle and the tibia tends to be pulled backward when a patient assumed supine position(11), the graft would have been suffering impaired strength leading to joint laxity. Fourthly, the diameter of the hamstring muscle also exerts influence on development of the secondary laxity after PCL reconstruction. According to literature, the average diameter of PCL was 10$11 \mathrm{~mm}(17,18)$ and the bony tunnel for single-strand PCL reconstruction of 9.5-11.5 mm (mean: 
$10.5 \mathrm{~mm}$ ) was recommended (19). In this study, the average diameter of the four-strand hamstring tendon was $7.11 \mathrm{~mm}$, which was far less than the actual diameter of PCL. A thinner tendon is unable to afford sufficient strength and tenacity, which raised the long-term risk of ligament laxity $(20,21)$.

Compared with HT group, PCL reconstruction with LARS could achieve early stability and is especially suitable for those who wish to resume sports activities earlier since, with LARS reconstruction, patients could engage in rehabilitative exercises earlier, which reduces the possibility of synarthrophysis $(22,23)$.

Our study also found that the diameter of the four-strand hamstring tendon was positively correlated with height and bore no relationship with body weight and BMI, and the hamstring tendon diameter was greater in males than in females, which were in agreement with the results reported by other researchers(21). Therefore, for female patients or patients who are physically shorter, the risk of operative failure related to a thinner hamstring tendon should be taken into account and relevant measures should be taken to address such risk in terms of graft selection and operative technique.

This study had some limitations. Firstly, to minimize bias, we established strict exclusion criteria. As a result, the sample size was relatively small. Secondly, the development of laxity secondary to PCL reconstruction with autologous tendon is multifactorial, involving operative skill, graft fixation, location of bony tunnel, killer turn and so on. Nonetheless, we made an effort to eliminate the aforementioned factors, including using the same fixation strategy, performing the operation by the doctors of the same team. Though the sample size was small, the results are, to some extent, of reference significance. Thirdly, although the subjective scores of two groups were similar in mid-term follow-up, their results of long term follow-up might be different because patients in LARS group attained better knee stability. It needs longer follow-up to confirm.

\section{Conclusion}

Both LARS and hamstring tendon autograft could have good efficacy for PCL reconstruction. The subjective scores of two groups were similar at the last follow-up, but patients in LARS group attained better knee stability. LARS, as an alternative grafts for PCL reconstruction, is especially suitable for those who hope to resume activities as early as possible.

\section{Declarations}

Ethics approval and consent to participate The study was approved by the Ethics Committee of the PLA General Hospital.Informed consent was obtained from all individual participants included in the study.

Consent for publication The participant has consented to the submission of the case report to the journal.

Availability of data and materials The datasets used and/or analysed during the current study are available from the corresponding author on reasonable request. 
Competing interests The authors declare that they have no competing interests

Funding No funding was received.

Authors' contributions All authors contributed to the study conception and design. Material preparation, data collection and analysis were performed by Yang Luo and Zhijiang Li. The first draft of the manuscript was written by Yang Luo. Zhigang Wang and Min Wei contributed to the revision.All authors commented on previous versions of the manuscript. All authors read and approved the final manuscript.

Acknowledgements Not applicable.

\section{References}

1. Fanelli GC, Edson CJ. Posterior cruciate ligament injuries in trauma patients: Part II. Arthroscopy: the journal of arthroscopic related surgery : official publication of the Arthroscopy Association of North America the International Arthroscopy Association. 1995;11(5):526-9.

2. Fanelli GC, Beck JD, Edson CJ. Current concepts review: the posterior cruciate ligament. J Knee Surg. 2010;23(2):61-72.

3. Panigrahi R, Kumari Mahapatra A, Priyadarshi A, Singha Das D, Palo N, Ranjan Biswal M. Outcome of Simultaneous Arthroscopic Anterior Cruciate Ligament and Posterior Cruciate Ligament Reconstruction With Hamstring Tendon Autograft: A Multicenter Prospective Study. Asian journal of sports medicine. 2016;7(1):e29287.

4. Sun K, Zhang J, Wang Y, Xia C, Zhang C, Yu T, et al. Arthroscopic reconstruction of the anterior cruciate ligament with hamstring tendon autograft and fresh-frozen allograft: a prospective, randomized controlled study. Am J Sports Med. 2011;39(7):1430-8.

5. Chen CP, Lin YM, Chiu YC, Wu HW, Lee CH, Tong KM, et al. Outcomes of arthroscopic double-bundle PCL reconstruction using the LARS artificial ligament. Orthopedics. 2012;35(6):e800-6.

6. Gliatis J, Anagnostou K, Tsoumpos P, Billis E, Papandreou M, Plessas S. Complex knee injuries treated in acute phase: Long-term results using Ligament Augmentation and Reconstruction System artificial ligament. World journal of orthopedics. 2018;9(3):24-34.

7. Saragaglia D, Francony F, Gaillot J, Pailhé R, Rubens-Duval B, Lateur G. Posterior cruciate ligament reconstruction for chronic lesions: clinical experience with hamstring versus ligament advanced reinforcement system as graft. International orthopaedics. 2020;44(1):179-85.

8. Canale ST, Beaty HJ. Campbell's Operative Orthopaedics. 11, editor: Elsevier,Singapore.; 2008.

9. Xu X, Huang T, Liu Z, Wen H, Ye L, Hu Y, et al. Hamstring tendon autograft versus LARS artificial ligament for arthroscopic posterior cruciate ligament reconstruction in a long-term follow-up. Arch Orthop Trauma Surg. 2014;134(12):1753-9.

10. Mardani-Kivi M, Karimi-Mobarakeh M, Keyhani S, Saheb-Ekhtiari K, Hashemi-Motlagh K, Sarvi A. Hamstring tendon autograft versus fresh-frozen tibialis posterior allograft in primary arthroscopic 
anterior cruciate ligament reconstruction: a retrospective cohort study with three to six years followup. International orthopaedics. 2016;40(9):1905-11.

11. Jung YB, Tae SK, Lee YS, Jung HJ, Nam CH, Park SJ. Active non-operative treatment of acute isolated posterior cruciate ligament injury with cylinder cast immobilization. Knee surgery, sports traumatology, arthroscopy: official. journal of the ESSKA. 2008;16(8):729-33.

12. Gao K, Chen S, Wang L, Zhang W, Kang Y, Dong Q, et al. Anterior cruciate ligament reconstruction with LARS artificial ligament: a multicenter study with 3- to 5-year follow-up. Arthroscopy: the journal of arthroscopic related surgery : official publication of the Arthroscopy Association of North America the International Arthroscopy Association. 2010;26(4):515-23.

13. Ahn JH, Wang JH, Lee YS, Kim JG, Kang JH, Koh KH. Anterior cruciate ligament reconstruction using remnant preservation and a femoral tensioning technique: clinical and magnetic resonance imaging results. Arthroscopy: the journal of arthroscopic related surgery : official publication of the Arthroscopy Association of North America the International Arthroscopy Association. 2011;27(8):1079-89.

14. Li B, Wen Y, Wu H, Qian Q, Wu Y, Lin X. Arthroscopic single-bundle posterior cruciate ligament reconstruction: retrospective review of hamstring tendon graft versus LARS artificial ligament. International orthopaedics. 2009;33(4):991-6.

15. Janssen RP, Scheffler SU. Intra-articular remodelling of hamstring tendon grafts after anterior cruciate ligament reconstruction. Knee surgery, sports traumatology, arthroscopy:. official journal of the ESSKA. 2014;22(9):2102-8.

16. Marumo K, Saito M, Yamagishi T, Fujii K. The "ligamentization" process in human anterior cruciate ligament reconstruction with autogenous patellar and hamstring tendons: a biochemical study. Am J Sports Med. 2005;33(8):1166-73.

17. Girgis FG, Marshall JL, Monajem A. The cruciate ligaments of the knee joint. Anatomical, functional and experimental analysis. Clinical orthopaedics and related research. 1975(106):216-31.

18. Tao C, He A, Wang W. Anatomy and clinical value of the posterior cruciate ligament of knee joint. Chinese Journal of Clinical anatomy. 2007;25(02):176-8.

19. Chen L, Yu J, Ao Y, Wang H, Lin L. Morphological Measurement of PCL Insertion and Optimized Tunnel Diameter for PCL Reconstruction. Chin J Sports Med. 2011;30(10):911-5 + 47.

20. Li B, Wang JS, He M, Wang GB, Shen P, Bai LH. Comparison of hamstring tendon autograft and tibialis anterior allograft in arthroscopic transtibial single-bundle posterior cruciate ligament reconstruction. Knee surgery, sports traumatology, arthroscopy: official journal of the ESSKA. 2015;23(10):3077-84.

21. Conte EJ, Hyatt AE, Gatt CJ Jr, Dhawan A. Hamstring autograft size can be predicted and is a potential risk factor for anterior cruciate ligament reconstruction failure. Arthroscopy: the journal of arthroscopic related surgery : official publication of the Arthroscopy Association of North America the International Arthroscopy Association. 2014;30(7):882-90. 
22. Bianchi N, Sacchetti F, Bottai V, Gesi M, Carlisi A, Facchini A, et al. LARS versus hamstring tendon autograft in anterior cruciate ligament reconstruction: a single-centre, single surgeon retrospective study with 8 years of follow-up. European journal of orthopaedic surgery traumatology: orthopedie traumatologie. 2019;29(2):447-53.

23. LI Y, Zhang H, Xiao S, Zheng Q, Zeng Y, Yang H. Effectiveness comparison of LARS artificial ligament and autogenous hamstring tendon in one-stage reconstruction of anterior and posterior cruciate ligaments. Chinese Journal of Reparative Reconstructive Surgery. 2020;34(5):1-7.

\section{Tables}

Table 1 Patients characteristics of LARS group and HT group

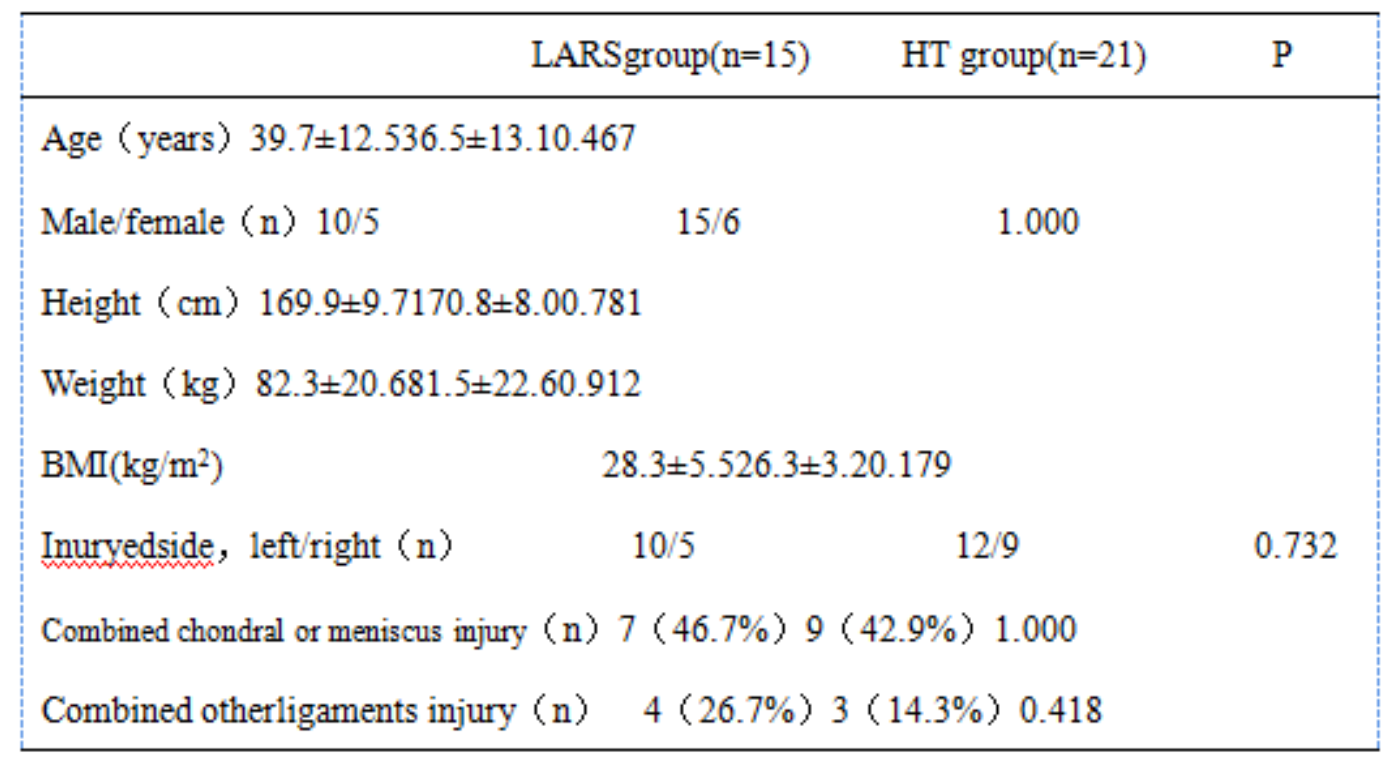


Table 2 Subjective function scores between the two groups

\begin{tabular}{|c|c|}
\hline LARSgroup $(n=15)$ & HTgroup(n=21)P \\
\hline \multicolumn{2}{|l|}{ Lysholm } \\
\hline \multicolumn{2}{|l|}{ Preoperation $44.9 \pm 22 \cdot 139.8 \pm 17.30 .438$} \\
\hline \multicolumn{2}{|l|}{6 month $78.6 \pm 11.270 .0 \pm 11.20 .030^{*}$} \\
\hline \multicolumn{2}{|l|}{12 month85.3 $311.182 .1 \pm 11.30 .399$} \\
\hline \multicolumn{2}{|l|}{ Final Follow-ups9.0 $\pm 9.985 .7 \pm 11.70 .377$} \\
\hline \multicolumn{2}{|l|}{ IKDC subjective } \\
\hline \multicolumn{2}{|l|}{ Preoperation $37.1 \pm 16.832 .8 \pm 14.70 .426$} \\
\hline \multicolumn{2}{|l|}{ 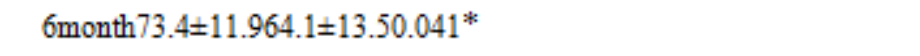 } \\
\hline \multicolumn{2}{|l|}{12 month $78.4 \pm 11.275 .3 \pm 10.90 .414$} \\
\hline \multicolumn{2}{|l|}{ Final Follow-up $81.1 \pm 10.780 .5 \pm 11.10 .873$} \\
\hline \multicolumn{2}{|l|}{ Tegner } \\
\hline \multicolumn{2}{|l|}{ Preoperation $1.4 \pm 0.81 .3 \pm 0.90 .817$} \\
\hline Final Follow-up $4.9 \pm 1.34 .5 \pm 1.40 .339$ & \\
\hline
\end{tabular}

$* \mathrm{P}<0.05$

Table 3 Knee joint stability between the two groups

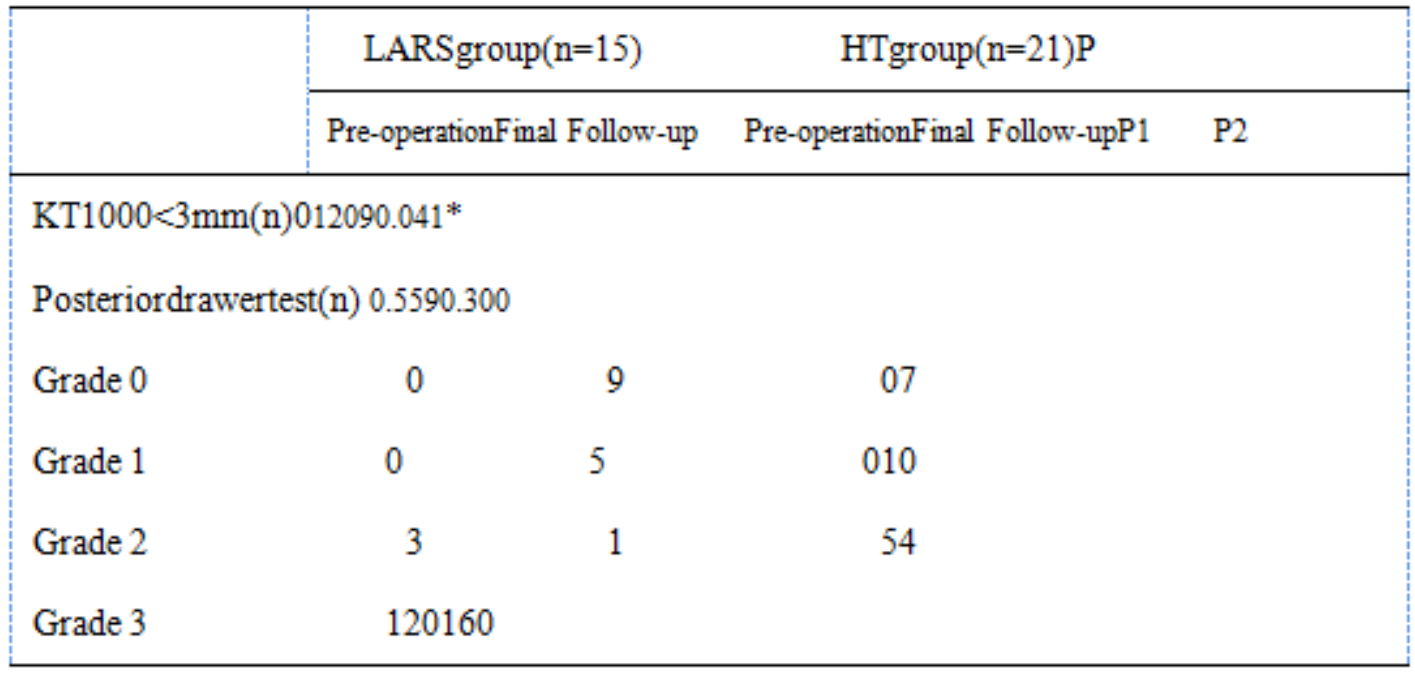

P1:pre-operative values, LARS group vs. HT group; P2:post-operative values, LARS group vs. HT group.

$* \mathrm{P}<0.05$

Figures 


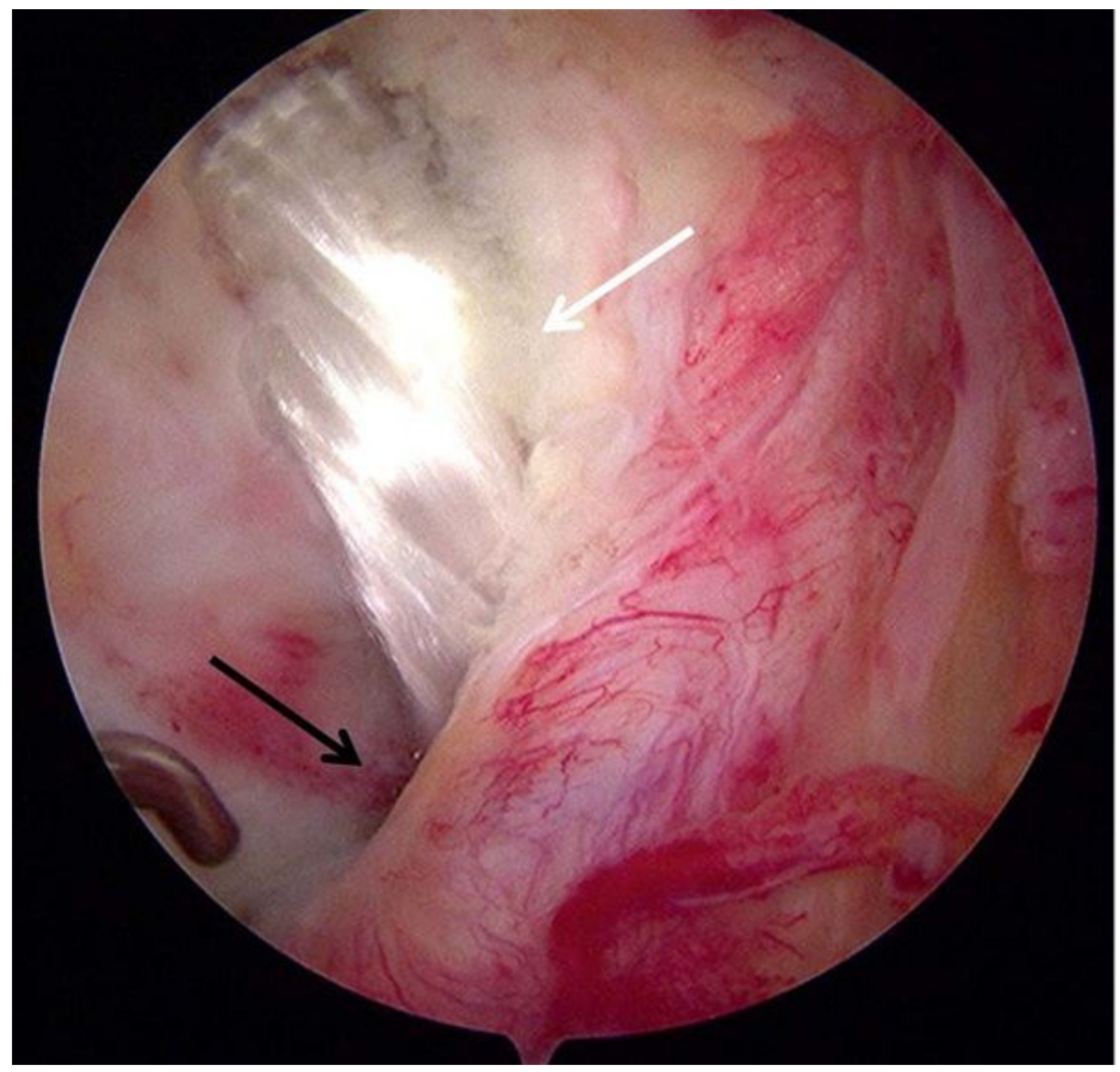

Figure 1

LARS group.The ACL (black arrow) and reconstructed PCL (white arrow) under arthroscopy 


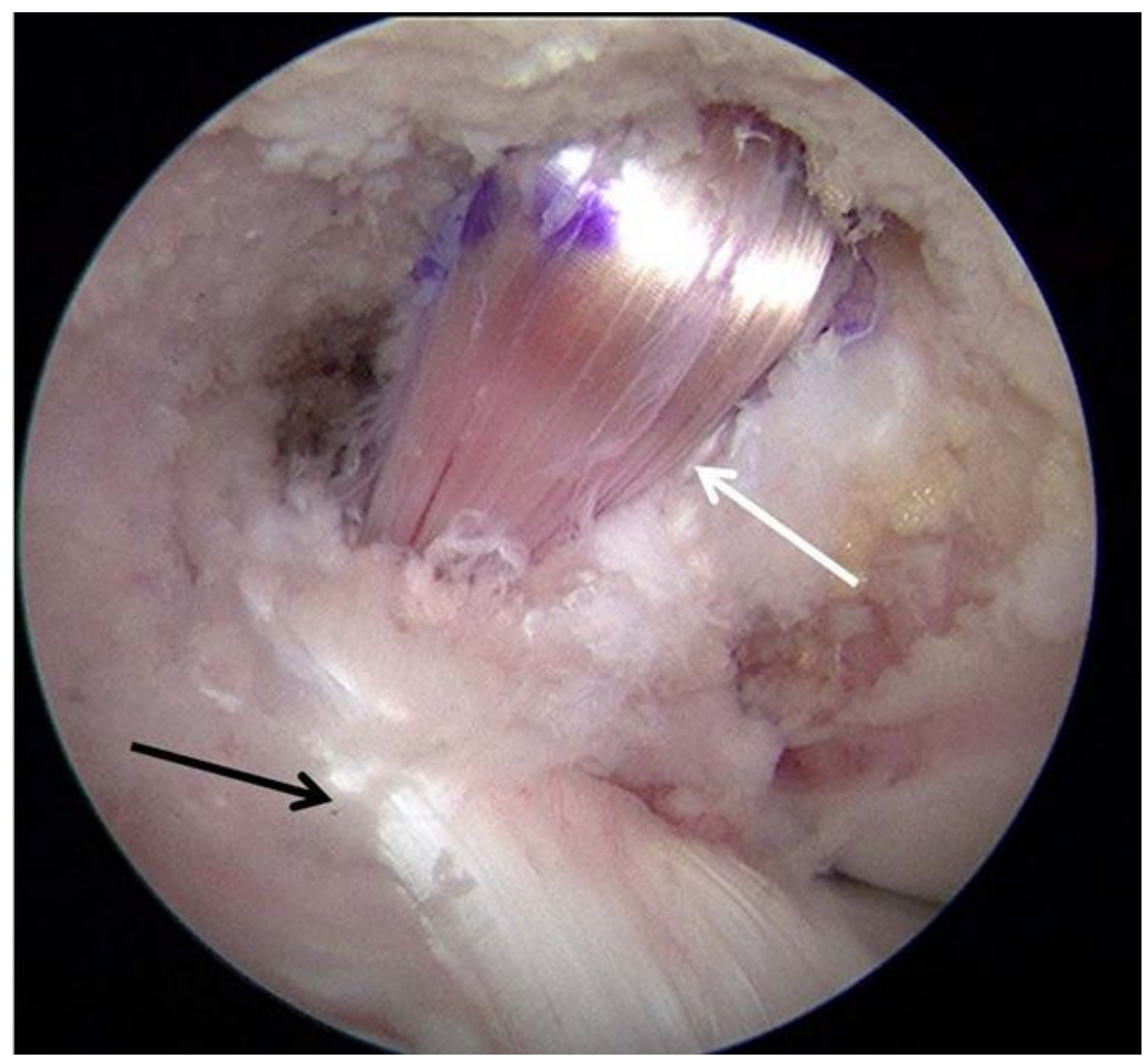

Figure 2

HT group.The ACL (black arrow) and reconstructed PCL (white arrow) under arthroscopy 